\title{
A ansiedade e o distanciamento familiar em estudantes universitários
}

\author{
Anxiety and family distancing in university students
}

Ansiedad y distanciamiento familiar en estudiantes universitarios

Luziaylla Alves Santos ${ }^{1 *}$, lasmin Santos Andrade ${ }^{2}$, Thatiane Andrade Souza ${ }^{2}$, lane Brito Leal ${ }^{2}$, Simone Yuriko Kameo ${ }^{2}$, Simone Otilia Cabral Neves², Magna Galvão Peixoto².

\section{RESUMO}

Objetivo: Analisar a correlação entre ansiedade e o distanciamento familiar em estudantes da Universidade Federal de Sergipe (UFS) - campus Lagarto. Métodos: Trata-se de uma pesquisa descritiva e quantitativa realizada com discentes da Universidade Federal de Sergipe campus de Lagarto. A pesquisa foi realizada nos meses de junho e julho de 2019, em que se utilizou o Inventário de Ansiedade de Beck (BAI) para medir a intensidade dos sintomas da ansiedade por meio do auto relato. Resultados: Dados de 145 universitários foram coletados. Desses, $62,8 \%$ eram do sexo feminino, 94,5\% tinham entre 18 e 21 anos de idade, $69 \%$ eram solteiros (as), 95,2\% não possuíam vínculo empregatício, 60,7\% moravam na cidade onde a universidade se localiza, 58,6\% eram sedentários, 81,4\% dormiam menos que 7 horas, $11,7 \%$ possuíam diagnóstico de doença mental, $76,6 \%$ não realizavam tratamento psicológico e $25,5 \%$ usavam medicamentos para a ansiedade. Houve homogeneidade na quantidade de casos para a ansiedade leve, moderada e grave, com menores casos de ansiedade mínima, sendo que esses níveis se relacionaram significativamente apenas com realizar atividade física $(0,019)$ e tratamento com psicólogo $(0,045)$. Conclusão: Há alta prevalência de ansiedade entre os universitários estudados, porém esse fato não está relacionado ao distanciamento familiar.

Palavras-chave: Ansiedade, Estudantes, Adulto jovem, Família.

\section{ABSTRACT}

Objective: Analyze the correlation between anxiety and family distance in students at the Federal University of Sergipe (UFS) - Lagarto campus. Methods: This is a descriptive and quantitative research carried out with students from the Federal University of Sergipe campus of Lagarto. The research was developed in June and July, 2019, the Beck Anxiety Inventory (BAl) was used to measure the intensity of anxiety symptoms through self-report. Results: Data from 145 university students were collected. Of these, 62,8\% were female, $94,5 \%$ were between 18 and 21 years old, $69 \%$ were single, $95,2 \%$ had no employment, $60,7 \%$ lived in the city where the university is located, $58,6 \%$ were sedentary, $8,4 \%$ slept less than 7 hours, $11,7 \%$ had a diagnosis of mental illness, $76,6 \%$ did not underwent psychological treatment and $25,5 \%$ used medication for anxiety. There was homogeneity in the number of cases for mild, moderate and severe anxiety, with lower cases of minimal anxiety, and these levels were significantly related only to physical activity $(0,019)$ and treatment with a psychologist $(0,045)$. Conclusion: There is a high prevalence of anxiety among the university students in this research, but this fact is not related to family distance.

Keywords: Anxiety, Students, Young adult, Family.

\section{RESUMEN}

Objetivo: Analizar la correlación entre ansiedad y distanciamiento familiar en estudiantes de la Universidad Federal de Sergipe (UFS) - campus Lagarto. Métodos: Se trata de una investigación descriptiva y cuantitativa realizada con estudiantes de la Universidad Federal de Sergipe campus Lagarto. La investigación se llevó a cabo en los meses de junio y julio de 2019, en lo que se utilizó el Inventario de Ansiedad de Beck (BAI) para medir la intensidad de los síntomas de ansiedad a través del auto informe. Resultados: Se recolectaron datos de 145 estudiantes universitarios. De estos, $62,8 \%$ eran mujeres, $94,5 \%$ tenían entre 18 y 21 años de edad, $69 \%$ eran solteros, $95,2 \%$ no tenían empleo, 60,7\% vivían en la ciudad donde se ubica la universidad, 58,6\% eran sedentarios, 81,4\% dormían menos de 7 horas, $11,7 \%$ tenían diagnóstico de enfermedad mental, $76,6 \%$ no se sometieron al tratamiento

\footnotetext{
${ }^{1}$ Universidade Federal de Sergipe (UFS), São Cristóvão - SE. *E-mail: luziayllasantos@gmail.com
}

2 Universidade Federal de Sergipe (UFS), Lagarto - SE. 
psicológico y $25,5 \%$ usaban medicinas para la ansiedad. Hubo homogeneidad en el número de casos de ansiedad leve, moderada y severa, con menores casos de ansiedad mínima, y estos niveles se relacionaron significativamente solo con la actividad física $(0,019)$ y el tratamiento con psicólogo $(0,045)$. Conclusión: Hay una alta prevalencia de ansiedad entre los estudiantes universitarios escuestados, pero este hecho no se relaciona conel familiar.

Palabras clave: Ansiedad, Estudiantes, Adulto joven, Família.

\section{INTRODUÇÃO}

A ansiedade caracteriza-se por sentimentos de tensão e de medo provocados pela sensação desagradável e desconcertante de apreensão, antecipação de algo desconhecido que ocorrerá futuramente, ou seja, pensamentos preocupantes (MOURA IMA, et al., 2018). Os sintomas podem ser físicos (palpitações, agitação, transpiração, problemas temporários na dicção) e psicológicos (medo de ser avaliado negativamente, pessimismo) (APA, 2014).

A ansiedade pode ser ainda caracterizada de duas maneiras: a ansiedade-estado que é uma emoção momentânea devido a uma situação específica e a ansiedade-traço que é uma característica intrínseca do indivíduo e o acompanha em diversas situações de uma maneira estável (CORDEIRO RA e FREIRE V, 2016).

Um acontecimento marcante e que demostra claramente os sintomas da ansiedade é a realização de alguma prova ou exame que teste a capacidade de um estudante, em que pode haver autocobrança, sentimento de incapacidade, nervosismo e outros sentimentos ruins. De forma geral, a ansiedade não se restringe somente ao exato momento da realização da prova, mas ao antes e ao depois. (GONZAGA et al., 2016).

A ansiedade pode ter antecedentes genéticos, psicológicos e socais. O genético relaciona-se a um conjunto de genes que são expressados de acordo com o aparecimento de fatores ambientais ou sociais. Como fator psicológico cita-se a ação acumulativa de eventos na infância que estão bastante associados a crianças privadas de eventos estressores por causa de pais superprotetores, por isso são mais propensas a serem ansiosas futuramente.

Por fim há os eventos estressores de natureza social e interpessoal que são: relacionamentos afetivos, dificuldades no trabalho, morte de um parente, pressões acadêmicas, entre outros (BARLOW DH e DURAND VM, 2012).

No meio acadêmico, a prevalência de ansiedade é significativamente grande em que, segundo estudo, $64,7 \%$ dos estudantes amostrais de uma universidade apresentavam algum nível de ansiedade (BENTO LMA, et al., 2017). Outro quesito importante é a maior prevalência da ansiedade em mulheres por causa de sua necessidade em superar mais obstáculos impostos pela sociedade que ainda é muito machista (BORINE RCC, et al., 2015)

Nessa fase da vida, os universitários estão expostos a várias modificações causadoras de estresse e ansiedade que podem interferir no bom desenvolvimento acadêmico e na sua qualidade de vida (LANTYER AS, 2016). É exigido do universitário uma mudança emocional e de percepção do meio para se adaptar à nova conjuntura de vida e aos requisitos para obterem a sua formação (CHAVES ECL, et al., 2015).

Além de que, no contexto atual das tecnologias, a demanda para o mercado de trabalho é de pessoas proativas, criativas, com a capacidade de (re) inventar e flexíveis a rápidas mudanças (FERREIRA BC, et al., 2014).

Como dito, boa parte dos estudantes apresentam algum nível de ansiedade, mas uma parcela pode ter níveis mais elevados: a porção que se desloca espacialmente e, consequentemente, distanciam-se do seio familiar. A falta da família como sistema protetor influencia na saúde física, mental e, principalmente, na emocional, uma vez que essa é uma instituição com bastante peso na formação de uma pessoa (ALMEIDA JSP, 2014). 
A saída de seu local de origem e ambiente físico, ou seja, esse distanciamento geográfico pode gerar o homesickness, as saudades de casa, que pode impor dificuldades de adaptação da nova conjuntura de vida do indivíduo, como a própria mudança de ambiente e o estabelecimento de novos papeis para uma nova rede social e cultural (OSSE CMC e COSTA II, 2011; SILVA SIC, 2013).

Nesse contexto, foram avaliados alguns parâmetros para escolha do tema como a carência de estudos relacionando ansiedade e família no campus universitário estudado. Além disso, consideramos que, por ser uma universidade localizada numa cidade do interior do estado de Sergipe, muitos universitários vêm de fora e se distanciam de seu núcleo familiar, trazendo consigo vulnerabilidade emocional e física resultando, assim, em ansiedade.

\section{MÉTODOS}

Trata-se de uma pesquisa descritiva, quantitativa, realizada com discentes da Universidade Federal de Sergipe, campus Prof. Antônio Garcia Filho, localizada na cidade de Lagarto, dos oito cursos ofertados neste campus: enfermagem, farmácia, fisioterapia, fonoaudiologia, medicina, nutrição, odontologia e terapia ocupacional.

A pesquisa foi realizada nos meses de junho e julho de 2019. Foram incluídos alunos do ciclo comum (primeiro ano) que frequentam a universidade e que concordaram em participar do estudo ao assinar o termo de consentimento livre e esclarecido (TCLE).

Foram excluídos os alunos que não frequentaram as aulas, os que já cursaram algum curso do ensino superior, além dos menores de 18 anos. A coleta de dados foi feita por meio do instrumento Inventário de Ansiedade de Beck - BAI, além de um questionário sociodemográfico adicionado para que fosse possível alcançar o objetivo proposto.

O inventário foi criado por Beck em 1988 para medir a intensidade dos sintomas da ansiedade por meio do auto relato (BECK AT, et al., 1988). Ele foi adaptado para o português e validado por Cunha em 2001 e é aplicado a população em geral.

É composto por 21 itens que podem ser respondidos de quatro maneiras: 0 - Absolutamente não; 1 Levemente; 2 - Moderadamente; 3 - Gravemente. O escores são: 0 a 10 - Sintomas mínimos; 11 a 19 Sintomas leves; 20 a 30 - Sintomas moderados; 31 a 63 - Sintomas graves; (CUNHA JA, 2001).

A pesquisa foi autorizada pelo comitê de ética em pesquisa da Universidade Federal de Sergipe, sob o número CAAE 08878319.7.0000.5546. Todos os participantes ficaram cientes em relação ao objetivo da pesquisa, além de terem participação voluntária em que a desistência pode ocorrer a qualquer momento, sendo garantido anonimato.

A pesquisa está de acordo com os princípios éticos de não maleficência, beneficência, justiça e autonomia, abordados na Resolução № 466, de 12 de dezembro de 2012 pelo Conselho Nacional de Saúde (BRASIL, 2012). Os dados foram tabulados e analisados no Software Microsoft Office Excel.

\section{RESULTADOS E DISCUSSÃO}

Foram coletados dados de 145 discentes que cursavam o primeiro ano em uma universidade de ensino superior de oito cursos diferentes na área da saúde (farmácia, fisioterapia, medicina, odontologia, enfermagem, terapia ocupacional, nutrição e fonoaudiologia).

As maiores frequências encontradas entre as caraterísticas sociodemográficas são descritas a seguir. Entre os estudantes pesquisados, 91 (62,8\%) eram do sexo feminino, 94,5\% dos estudantes tinham entre 18 e 21 anos de idade, 69\% eram solteiros (as). Em relação a cor, a cor parda predominou (64,8\%).

A maioria relatou ser estudante e não possuir vínculo empregatício (95,2\%), 88 (60,7\%) moram na cidade onde a universidade se localiza. Em relação a prática de atividade física, o sedentarismo predominou $(58,6 \%)$ e a maioria relata ter menos que 7 horas de sono $(81,4 \%)$. 
Se tratando de diagnóstico de psiquiátrico, 17 (11,7\%) afirmaram possuir diagnóstico de algum tipo de transtorno psiquiátrico e $128(88,3 \%)$ não apresentaram. Os estudantes que não fazem terapia psicológica para ansiedade $(76,6 \%)$ representam a maioria, ao passo que poucos $(23,4 \%)$ realizam tratamento com psicólogo. Trinta e sete estudantes (25,5\%) fazem uso de medicamentos para ansiedade e 108 (74,5\%) não fazem utilização.

Os cursos de terapia ocupacional e nutrição apresentaram níveis de ansiedade mais elevados, enquanto que o curso de medicina foi o que apresentou a menor quantidade de casos graves. Diferentemente deste estudo, o estudo de Leão AM, et al. (2018) demostrou que o curso de fisioterapia seguido pelo de odontologia possuem maior prevalência de ansiedade, que corrobora em parte com o estudo de Ferreira BC, et al. (2019) em que psicologia segue em primeiro e fisioterapia em segundo.

É interessante observar que os dados retratam uma realidade diferente da esperada, em que o curso de medicina apresentaria casos mais graves de ansiedade. A carga horária e a grade curricular com dedicação integral do curso de medicina podem desencadear situações de estresse, ansiedade e depressão, principalmente, pelo pouco tempo dedicado a atividades de lazer. Sendo que $47,6 \%$ dos participantes de uma pesquisa relataram aproveitar a vida mais ou menos, muito pouco ou nada, ou seja, não aproveitam completamente a juventude (BAMBI LNS, et al., 2013).

Dos indivíduos que responderam o BAl, o menor escore encontrado foi 0 e o maior foi 53. Quanto ao grau de ansiedade, o presente estudo mostrou uma homogeneidade na quantidade de casos para a ansiedade leve, moderada e grave, com menores casos de ansiedade mínima, mostrando que a maior parte dos estudantes universitários tem níveis de ansiedade mais elevados, enquanto que uma minoria tem níveis que seriam considerados normais (Tabela 1).

Em contrapartida, outro estudo observou muito mais casos de estudantes com grau mínimo $(37,1 \%)$ e grau leve $(30,2 \%)$ de ansiedade, ao passo que o grau grave $(11,2 \%)$ apresentou pouca frequência (FERNANDES MA, et al., 2018).

Tabela 1 - Grau de ansiedade dos universitários da saúde que cursam o primeiro ano.

\begin{tabular}{lll}
\hline Variável & N & $\%$ \\
\hline Ansiedade mínima (0-10) & 30 & 20,7 \\
Ansiedade leve (11-19) & 39 & 26,9 \\
Ansiedade moderada (20-30) & 37 & 25,5 \\
Ansiedade grave (31-63) & 39 & 26,9 \\
\hline
\end{tabular}

Fonte: SANTOS LA, et al, 2020.

Diferentemente da maioria dos estudos encontrados, pudemos observar que a amostra deste estudo apresentou maior prevalência de casos mais graves de ansiedade. Em 2018, um estudo semelhante foi realizado na mesma universidade onde foram coletados os dados desta pesquisa.

Dos vinte estudantes de medicina do primeiro ano que foram abordados, 18 (90\%) apresentaram ansiedade mínima, enquanto que apenas 5 (23,8\%) estudantes apresentaram ansiedade mínima neste estudo, demostrando uma discrepância muito grande num estudo realizado apenas 1 ano depois (ROLLEMBERG GSM, 2018).

Diante disso, mesmo sendo apenas para os estudantes de medicina do primeiro ciclo, podemos abranger e fazer o questionamento de que algo ocorreu nesse período que não pudemos identificar e que causou, ou está causando, esse aumento na prevalência de ansiedade.

Um fator interessante para se abordar, quando se trata de estudos com universitários, seriam as diferentes prevalências de ansiedade durante os anos de curso, mas essa área não é muito relatada como sendo o foco das pesquisas, principalmente de forma longitudinal e detalhada.

O estudo de Lelis KC, et al (2020), por exemplo, fez a coleta dos dados com os estudantes dos diversos períodos letivos, mas não fez a relação com a prevalência de ansiedade. Já outros dois estudos relatam as prevalências de ansiedade para os diferentes anos de curso, mas sem apresentar o valor de significância dos cálculos estatísticos entre as variáveis (TAMY MSF, et al., 2018; LEITE BR, et al., 2020) 
Segundo o BAI, os cinco sintomas mais frequentes encontrados entre os universitários da área da saúde com casos graves de ansiedade em ordem decrescente de frequência foram: medo que aconteça o pior, nervoso, sem equilíbrio ou inseguro (a), palpitação ou aceleração no coração e incapaz de relaxar corroborando com o estudo de Andrade AM e Pires EU (2020). Enquanto que os sintomas menos frequentes foram: Sensação de desmaio, rosto afogueado (rubor facial), dormência ou formigamento, medo de morrer e sensação de calor.

O estudo de Fernandes MA, et al. (2018) mostra alguns dados semelhantes, em que os sintomas mais frequentes foram nervosismo, sensação de calor, medo que aconteça o pior, incapaz de relaxar e indigestão ou desconforto abdominal; e os menos frequentes foram dormência ou formigamento, sensação de desmaio, tremores nas pernas, sem equilíbrio, dificuldade de respirar e rosto afogueado.

No que se refere a moradia, $59(40,7 \%)$ moram com amigos e colegas ou sozinho (a), ao passo que 86 $(59,3 \%)$ moram com os pais ou familiares. Esses dados demostram que muitos estudantes saem de suas casas e do convívio familiar para estudar, porém, ao relacionar esse dado com os níveis de ansiedade, não houve relação significativa $(0,626)$.

No entanto, o estudo de Vizzotto MM, et al. (2017) mostrou uma relação significativa entre o nível de ansiedade e com quem o estudante mora, sendo que quem mora com pais ou familiares tem níveis menores de ansiedade, além de ter qualidade de vida melhor.

Um estudo desenvolvido por Flesch BD, et al. (2020) com universitários ingressantes do primeiro semestre encontrou uma maior prevalência de Episódio Depressivo Maior entre os estudantes que relataram morar com amigos ou colegas, o que pode ter relação com o distanciamento de suas famílias e com a dificuldade de adaptação a um novo ambiente.

Tal dificuldade de adaptação à moradia fora da família também foi observada no trabalho de Sousa LP (2020), em que um percentual significativo de estudantes de três universidades federais ressaltou alguns fatores prejudiciais como convivência com os demais residentes, queixas relacionadas à falta de privacidade, problemas de infraestrutura, situações de trote entre outros aspectos.

Apesar deste estudo não mostrar relação da ansiedade com o distanciamento familiar e de outros mostrarem tal relação, o estudo de Leão AM, et al. (2018) encontrou relação significativa entre ansiedade e relacionamento insatisfatório do estudante com familiares, amigos e colegas, demonstrando que independentemente de quem seja, a prevalência de ansiedade está relacionada ao fator da convivência social.

A autora cita essa relação como algo que é intrínseco ao sistema social, demonstrando a necessidade de equilíbrio em todas as esferas da vida do estudante, pois, sem esse equilíbrio, o estudante acaba tendo sua formação acadêmica prejudicada por conta de problemas emocionais e relacionais como baixa autoestima, preocupações, insegurança e dificuldades psicológicas.

De acordo com Osse CMC e Costa II (2011), a fase de vida entre 18 a 25 anos propicia o surgimento de comportamentos autodestrutivos, tais como o uso de álcool e de drogas, que pode ser sinalizado como necessidades de auxílio e apoio.

Mas eles têm dificuldades em buscar ajuda, e isso pode ser somado as mudanças ambientais, de rotina e nos sistemas de suporte social, como resultado do afastamento do ambiente familiar, dos amigos anteriores e a entrada no ensino superior. A entrada na universidade nem sempre significa estabilidade como desejado, pois os universitários podem manifestar algumas preocupações, dúvidas e ansiedade.

Foi analisada também a relação entre os valores do Inventário de Ansiedade de Beck com as demais variáveis. Não houve associação com idade $(0,313)$, sexo $(0,005)$, cor $(0,091)$, estado civil $(0,346)$, estudar e/ou trabalhar $(0,979)$, tipo de curso superior $(0,766)$, município que reside $(0,185)$, uso de medicamento para ansiedade $(0,061)$ e as horas de sono $(0,140)$. As únicas variáveis em que houve associação com nível de ansiedade foram realizar atividade física $(0,019)$ e tratamento com psicólogo $(0,045)$ (Tabela 2). 
Tabela 2 - Distribuição da intensidade dos sintomas de ansiedade dos universitários de acordo com as diversas variáveis, 2019.

\begin{tabular}{|c|c|c|c|c|c|c|c|c|c|c|}
\hline \multirow{3}{*}{ Variável } & & \multicolumn{8}{|c|}{ Intensidade dos sintomas de ansiedade } & \multirow{3}{*}{$\mathbf{P}$} \\
\hline & & \multicolumn{2}{|c|}{ Mínimo } & \multicolumn{2}{|c|}{ Leve } & \multicolumn{2}{|c|}{ Moderado } & \multicolumn{2}{|c|}{ Grave } & \\
\hline & & $\mathbf{N}$ & $\%$ & $\mathbf{N}$ & $\%$ & $\mathbf{N}$ & $\%$ & $\mathbf{N}$ & $\%$ & \\
\hline \multirow{3}{*}{ Sexo } & Não informado & 0 & 0 & 0 & 0 & 1 & 33,3 & 2 & 66,7 & \multirow{3}{*}{0,05} \\
\hline & Feminino & 11 & 12,1 & 24 & 26 & 27 & 29,7 & 29 & 31,9 & \\
\hline & Masculino & 19 & 37,3 & 15 & 29 & 9 & 17,6 & 8 & 15,7 & \\
\hline \multirow{5}{*}{ Idade } & Entre 18 e 19 anos & 21 & 18,9 & 33 & 30 & 29 & 26,1 & 28 & 25,2 & \multirow{5}{*}{0,313} \\
\hline & Entre 20 e 21 anos & 7 & 26,9 & 5 & 19 & 8 & 30,8 & 6 & 23,1 & \\
\hline & Entre 22 e 25 anos & 2 & 50 & 0 & 0 & 0 & 0 & 2 & 50 & \\
\hline & Entre 27 e 28 anos & 0 & 0 & 1 & 50 & 0 & 0 & 1 & 50 & \\
\hline & Entre 29 e 35 anos & 0 & 0 & 0 & 0 & 0 & 0 & 2 & 100 & \\
\hline \multirow{6}{*}{ Cor } & Parda & 15 & 16 & 29 & 31 & 20 & 21,3 & 30 & 31,9 & \multirow{6}{*}{0,091} \\
\hline & Branca & 9 & 27,3 & 6 & 18 & 11 & 33,3 & 7 & 21,2 & \\
\hline & Amarela & 0 & 0 & 0 & 0 & 2 & 66,7 & 1 & 33,3 & \\
\hline & Preta & 6 & 46,2 & 4 & 31 & 3 & 23,1 & 0 & 0 & \\
\hline & Indígena & 0 & 0 & 0 & 0 & 0 & 0 & 1 & 100 & \\
\hline & Outros & 0 & 0 & 0 & 0 & 1 & 100 & 0 & 0 & \\
\hline \multirow{3}{*}{ Estado civil } & Não informado & 0 & 0 & 0 & 0 & 1 & 100 & 0 & 0 & \multirow{3}{*}{0,346} \\
\hline & Solteiro (a) & 18 & 18 & 25 & 25 & 26 & 26 & 31 & 31 & \\
\hline & Em relacionamento & 12 & 27,3 & 14 & 32 & 10 & 22,7 & 8 & 18,2 & \\
\hline \multirow{2}{*}{ Estuda/Trabalha } & Estuda & 29 & 21 & 37 & 27 & 35 & 25,4 & 37 & 26,8 & \multirow{2}{*}{0,979} \\
\hline & Trabalha & 1 & 14,3 & 2 & 29 & 2 & 28,6 & 2 & 28,6 & \\
\hline \multirow{8}{*}{ Curso } & Enfermagem & 3 & 20 & 4 & 27 & 3 & 20 & 5 & 33,3 & \multirow{8}{*}{0,766} \\
\hline & Farmácia & 3 & 12,5 & 8 & 33 & 8 & 33,3 & 5 & 20,8 & \\
\hline & Fisioterapia & 4 & 18,2 & 5 & 23 & 8 & 36,4 & 5 & 22,7 & \\
\hline & Fonoaudiologia & 2 & 14,3 & 2 & 14 & 6 & 42,9 & 4 & 28,6 & \\
\hline & Medicina & 5 & 23,8 & 7 & 33 & 5 & 23,8 & 4 & 19 & \\
\hline & Nutrição & 4 & 26,7 & 5 & 33 & 0 & 0 & 6 & 40 & \\
\hline & Odontologia & 5 & 26,3 & 6 & 32 & 4 & 21,1 & 4 & 21,1 & \\
\hline & $\begin{array}{l}\text { Terapia } \\
\text { ocupacional }\end{array}$ & 4 & 26,7 & 2 & 13 & 3 & 20 & 6 & 40 & \\
\hline \multirow{3}{*}{ Local de residência } & Não informado & 1 & 50 & 1 & 50 & 0 & 0 & 0 & 0 & \multirow{3}{*}{0,185} \\
\hline & $\begin{array}{l}\text { Dentro da cidade } \\
\text { que estuda }\end{array}$ & 19 & 21,6 & 28 & 32 & 23 & 26,1 & 18 & 20,5 & \\
\hline & $\begin{array}{l}\text { Fora da cidade que } \\
\text { estuda }\end{array}$ & 10 & 18,2 & 10 & 18 & 14 & 25,5 & 21 & 38,2 & \\
\hline \multirow{3}{*}{ Com quem reside } & Amigos ou colegas & 13 & 28,9 & 10 & 22 & 13 & 28,9 & 9 & 20 & \\
\hline & Pais ou familiares & 14 & 16,7 & 24 & 29 & 21 & 25 & 25 & 29,8 & 0,626 \\
\hline & Sozinho (a) & 3 & 18,8 & 5 & 31 & 3 & 18,8 & 5 & 31,3 & \\
\hline Prática de atividade & Sim & 19 & 31,7 & 16 & 27 & 15 & 25 & 10 & 16,7 & 0.019 \\
\hline física & Não & 11 & 12,9 & 23 & 27 & 22 & 25,9 & 29 & 34,1 & \\
\hline $\mathrm{Ho}$ & Menos de 7 horas & 24 & 20,3 & 28 & 24 & 30 & 25,4 & 36 & 30,5 & 014 \\
\hline noras & Mais de 7 horas & 6 & 22,2 & 11 & 41 & 7 & 25,9 & 3 & 11,1 & 0,14 \\
\hline & Sim & 5 & 13,5 & 7 & 19 & 9 & 24,3 & 16 & 43,2 & \\
\hline Uso de medicamentos & Não & 25 & 23,1 & 32 & 30 & 28 & 25,9 & 23 & 21,3 & 0,061 \\
\hline Tratamento & Sim & 4 & 11,8 & 6 & 18 & 9 & 26,5 & 15 & 44,1 & \\
\hline para ansiedade & Não & 26 & 23,4 & 33 & 30 & 28 & 25,2 & 24 & 21,6 & 0,045 \\
\hline
\end{tabular}

Legenda: $X^{2}$ de igualdade: $p<0,05$.

Fonte: Santos LA, et al., 2020. 
Em relação ao sexo, o feminino segue uma linearidade crescente quanto aos níveis de ansiedade, enquanto que o sexo masculino segue uma linearidade decrescente, porém, por muito pouco, esses dados não se mostraram significativos (0,005), assim como o estudo de Vizzotto $M M$, et al. (2017) que não apresentou relação entre sexo e ansiedade, somente para a depressão.

Apesar do achado neste estudo, a maior parte dos estudos publicados relatam que o sexo feminino tem maiores níveis de ansiedade (LEÃO AM, et al., 2018; COSTA DS, et al., 2020). Diante do atual sistema ainda machista, pode-se dizer que o sexo feminino ainda precisa quebrar mais barreiras sociais que os homens, e, como consequência, as mulheres tendem a realizar atividades em excesso como cuidar da casa, trabalhar ao mesmo tempo que estuda, entre outras situações (BORINE RCC, et al., 2015). Felizmente, segundo Andrade AM e Pires EU (2020), as mulheres se mostraram mais interessadas em procurar atendimento psicológico e, por isso, é bem justificável que elas tenham atendimento psicológico exclusivo dentro da universidade.

Para os universitários que fazem tratamento psicológico, $43,2 \%$ deles tem um grau grave de ansiedade, ao passo que apenas $21,3 \%$ de quem não faz tratamento tem grau grave. Isso é um dado interessante, pois mostra que os indivíduos mais ansiosos já procuraram algum tipo de tratamento, porém ainda não melhoraram, podendo indicar o tipo de ansiedade-traço.

Um outro fator associado a maiores níveis de ansiedade foi o sedentarismo. Nesse sentido, Toti TG, et al. (2018) relata que os estudantes praticantes de algum tipo de atividade física de lazer por mais de 150 minutos semanais são menos propensos a ter sinais de ansiedade. Esses dados só fortalecem a ideia já existente e comprovada de que a atividade física oferece maior qualidade de vida para os indivíduos, pois traz benefícios para o corpo, tanto fisiológicos, quanto espirituais e psicológicos.

Dentro do contexto diagnóstico de doenças psiquiátricas algumas variáveis foram estudadas. Não foi observada associação do diagnóstico com sexo $(0,319)$, idade $(0,055)$, cor $(0,075)$, estado civil $(0,844)$, estudar e/ou trabalhar $(0,829)$, tipo de curso superior $(0,689)$, cidade que reside $(0,197)$, com quem reside $(0,650)$, praticar atividade física $(0,286)$ e as horas de sono $(0,440)$.

De acordo com as variáveis dicotômicas analisadas por meio do teste $X^{2} \mathrm{e}$ da razão de chance com os intervalos de confiança de $95 \%$, houve associação entre indivíduos que possuem diagnóstico de doenças psiquiátricas e fazem uso de medicação para ansiedade $(p=0,000)$. Sendo que indivíduos que têm diagnóstico confirmado possuem 9,8 (odds ratio $=9.888$ ) vezes mais chances em utilizar a medicação (intervalo de confiança entre 3.191 e 30.643). Além disto, universitários com diagnóstico confirmado têm 16,5 (odds ratio $=16.560)$ vezes mais chances em realizar tratamento com psicólogo $(p=0,000)$ (intervalo de confiança entre 4.916 e 55.775). Tais fatos são importantes, uma vez que as intervenções adotadas podem ajudar a minimizar os sinais e sintomas de doenças psiquiátricas.

Apesar do estudo não apresentar relação, outro estudo afirma que estrutura demográfica e social da atual população universitária sofreu mudanças importantes e são mais diversificadas, sobretudo em relação a idade, com uma variedade de estudantes entre 17 a 50 anos, em sua maioria do sexo feminino e com um aumento na faixa etária de 25-50 anos. Quanto às mudanças sociais, o desafio maior se dá para os estudantes mais velhos, pois reingressam na universidade entre a transição do trabalho para a faculdade, ou seja, eles precisam conciliar o trabalho com as exigências acadêmicas (PINTO JC, et al., 2016).

\section{CONCLUSÃO}

Com este estudo pode-se concluir que há uma alta prevalência de ansiedade entre os universitários estudados, porém esse fato não está relacionado ao distanciamento familiar vivenciado por alguns estudantes que saem de casa para estudar, como era esperado. Não realizar atividade física e fazer tratamento com psicólogo foram as únicas variáveis que estiveram atrelados a níveis maiores de ansiedade, ao passo que idade, sexo, cor, estado civil, estudar e/ou trabalhar, tipo de curso, cidade que o estudante reside e horas de sono não apresentaram relação significativa com qualquer nível de ansiedade. Apesar de alguns dados serem significativos, a amostra poderia ser maior, portanto, novos estudos podem ser realizados com um número maior de indivíduos, além de que mais variáveis podem ser adicionadas, tais como gênero, desempenho acadêmico, qualidade de vida, dentre outros. 


\section{AGRADECIMENTOS E FINANCIAMENTO}

Agradecemos a todos os estudantes que se disponibilizaram a fornecer os dados necessários para a realização da pesquisa, a Universidade Federal de Sergipe que aprovou esta pesquisa e aos professores da universidade que nos ajudaram cedendo espaço e tempo para que os acadêmicos pudessem responder os questionários. Não houve financiamento.

\section{REFERÊNCIAS}

1. ALMEIDA JSP. A saúde mental global, a depressão, a ansiedade e os comportamentos de risco nos estudantes do ensino superior: estudo de prevalência e correlação. Tese (Doutorado em Ciências da Vida, Especialidade em Saúde Mental) Faculdade de Ciências Médicas. 2014; 237 p.

2. ANDRADE AM, PIRES EU. Avaliação dos níveis de ansiedade dos estudantes da UFRRJ. Revista Trabalho (En)Cena, 2020; 5(1): 248-468.

3. AMERICAN PSYCHIATRIC ASSOCIATION. Manual Diagnóstico e Estatístico de Transtornos Mentais, DSM-5. 5 ed. Porto Alegre: Artmed, 2014; $991 \mathrm{p}$.

4. BAMPI LNS, et al. Qualidade de vida de estudantes de medicina da Universidade de Brasília. Revista Brasileira de educação médica, 2013; 37(2): 217-225.

5. BARLOW DH, DURAND VM. Abnormal psychology: an integrative approach. 6th ed. Wadsworth: Cengage Learning, 2012.

6. BECK AT, et al. An inventory for measuring clinical anxiety: psychometric properties. Journal of Consulting and Clinical Psychology, 1988; 56(6): 893-897.

7. BENTO LMA, et al. Percepção dos alunos de medicina quanto a aprendizagem $X$ ansiedade na metodologia ativa. Revista de Ensino, Educação e Ciências Humanas, 2017; 18(2): 178-182.

8. BORINE RCC, et al. Relação entre qualidade de vida e o estresse em acadêmicos da área da saúde. Estudos Interdisciplinares em Psicologia, 2015; 6(1): 100-118.

9. BRASIL. Ministério da saúde. Conselho Nacional de Saúde: Resolução no466. Brasília, 2012.

10. CHAVES ECL, et al. Ansiedade e espiritualidade em estudantes universitários: um estudo transversal. Revista Brasileira de Enfermagem (REBEn), 2015; 68(3): 504- 509.

11. CORDEIRO RA, FREIRE V. Estado-traço de ansiedade e vivências académicas em estudantes do $1^{\circ}$ ano do instituto politécnico de Portalegre. Revista Repositórios Científicos de Acesso Aberto de Portugal, 2016; 32(14): 1-7.

12. COSTA DS, et al. Sintomas de depressão, ansiedade e estresse em estudantes de medicina e estratégias institucionais de enfrentamento, 2020; 44(1): e040.

13. CUNHA JA. Manual das escalas em português das escalas de Beck. São Paulo: Casa do Psicólogo, $2001 ; 171$.

14. FERNANDES MA, et al. Prevalência de sintomas ansiosos e depressivos em universitários de uma instituição pública. Revista Brasileira de Enfermagem, 2018; 71(suppl 5): 2298-2304.

15. FERREIRA BC, et al. Verificação de ansiedade em acadêmicos dos cursos de saúde de uma universidade privada da zona da mata mineira. Interdisciplinary Scientific Journal, 2019; 6(5): 330-353.

16. FLESCH BD, et al. Episódio depressivo maior entre universitários do sul do Brasil. Revista de Saúde Pública, 2020; 54(31): $1-11$.

17. GONZAGA LRV, et al. Ansiedade de provas em estudantes do ensino médio. Revista Psicologia Argumento, 2016; 32(84): 76-88.

18. LANTYER $A$, et al. Ansiedade e qualidade de vida entre estudantes universitários ingressantes: avaliação e intervenção. Revista Brasileira de Terapia Comportamental e Cognitiva, 2016; 18(2): 4-19.

19. LEÃO AM, et al. Prevalência de fatores associados à depressão e ansiedade entre estudantes universitários da área da saúde de um grande centro urbano do nordeste do Brasil. Revista Brasileira de Educação Médica, 2018; 42(4): 55-65.

20. LEITE BR, et al. Associação entre qualidade de sono e ansiedade em acadêmicos de medicina. Brazilian Journal of Health Review, 2020; 3(3): 6528-6543.

21. LELIS KC, et al. Sintomas de depressão, ansiedade e uso de medicamentos em universitários. Revista Portuguesa de Enfermagem de Saúde Mental, 2020; (23): 9-14.

22. MOURA IMA, et al. Terapia cognitivo-comportamental no tratamento do transtorno de ansiedade generalizada. Revista científica da faculdade de educação e meio ambiente, 2018; 9(1): 423-441.

23. OSSE CMC, COSTA II. Saúde mental e qualidade de vida na moradia estudantil da Universidade de Brasília. Estudos de Psicologia, 2011; 28(1): 115-122.

24. PINTO JC, et al. Identificação de necessidades de intervenção psicológica: um estudo-piloto no ensino superior português. Psicologia USP, 2016; 27(3): 459-472.

25. ROLLEMBERG GSM. Avaliação da presença de sintomas de ansiedade e depressão em estudantes de medicina na Universidade Federal de Sergipe. Revista debates em psiquiatria, 2018; 8(3): 6-13.

26. SILVA SIC. Saudades de casa e estratégias de adaptação em estudantes em mobilidade internacional: determinantes e consequências. Dissertação (mestrado integrado em psicologia) - Faculdade de Medicina. Universidade de Lisboa, Lisboa, 2013; 81 p.

27. SOUSA, LP. A moradia estudantil no processo de afiliação e integração à vida acadêmica. Tese (Doutorado em Educação) - Faculdade de Educação da Universidade Federal de Minas Gerais, Belo Horizonte, 2020; 375 p.

28. TAMY MSF, et al. Cefaleia associada a indicadores de transtorno de ansiedade: Um estudo em acadêmicos de medicina. Interdisciplinary Scientific Journal, 2018; 5(5): 393-407.

29. TOTI TG, et al. Fatores associados à ansiedade e depressão em estudantes universitários do curso de educação física. Revista Saúde Física \& Mental, 2018; 6(2): 21-30.

30. VIZZOTTO MM, et al. Saudades de casa: indicativos de depressão, ansiedade, qualidade de vida de estudantes universitários. Revista Psicologia e Saúde, 2017; 9(1): 59-73. 